\title{
Relación entre el liderazgo transformacional y el desarrollo de equipos de alto rendimiento
}

\section{Relationship between transformational leadership and the development of high performance teams}

Lucía Magdalena Pico Versoza

Universidad Internacional del Ecuador, Ecuador

Ricardo Rafael Coello Yagual

Universidad Internacional del Ecuador, Ecuador

Autor para correspondencia: lupicove@ internacional.edu.ec; rcoelloy@ outlook.com

Fecha de recepción: 03 de mayo de 2018 - Fecha de aceptación: 30 de septiembre de 2018

\section{Resumen}

El presente estudio, busca analizar las principales características y relación que existe entre el liderazgo transformacional considerado como positivo hacia sus seguidores y, por la dinámica de mirar hacia fuera para la organización como un todo; siendo a su vez premisa que colabora en el desarrollo de equipos de alto rendimiento que buscan conducir sus objetivos hacia un mismo fin, es decir el éxito empresarial. Por lo cual, también se busca desarrollar los aspectos que permiten gestionar el cambio organizacional a través de las cualidades del líder dentro de los equipos que realizan la gestión organizacional.

Palabras Claves: liderazgo transformacional; organización; equipos de alto rendimiento

\begin{abstract}
The present study seeks to analyze the main characteristics and relationship that exists between the transformational leadership considered positive towards its followers and the dynamics of looking out for the organization as a whole; Being premise that collaborates in the development of teams of high performance that seek to lead their objectives towards the same end, that is to say, business success. Therefore, it also seeks to develop the aspects that allow management of organizational change through the qualities of the leader within the teams that perform organizational management.
\end{abstract}

Key words: transformational leadership; organization; teams of high performance 


\section{Introducción}

En la dinámica de mercados actuales, las empresas buscan constantemente talento humano capacitado y acorde al ritmo que desea llevar la empresa, sus esfuerzos se encuentran alineados al ritmo de la globalización, en donde los verdaderos responsables de la gestión de las organizaciones consideran: las competencias, las destrezas y habilidades de cada trabajador como cualidades para considerarlo como "apto", para formar parte del grupo de empleados que llevarán a la cima a la empresa (Pico, 2016), así también este grupo de individuos dentro de la organización necesitan la guía de parte de una persona, necesitan su dirección, su acompañamiento y la motivación constante para cumplir con los objetivos que plantea la organización, es indispensable comprender entonces la importancia de un líder que lleve el timón de la nave hacia un feliz puerto (Nader \& Castro, 2007).

Las compañías buscan el desarrollo de habilidades directivas dentro del talento humano que poseen, es indispensable la búsqueda continua del desarrollo de estas competencias para la obtención de resultados favorables a la organización. Siguiendo la línea de investigación analizaremos: ¿Qué hacen los directivos y qué habilidades necesitan para un trabajo efectivo? (Codina, 2017)

El liderazgo es una parte primordial en el desarrollo de equipos de alto rendimiento, así mismo lo tenemos definido como el proceso de influir, guiar y dirigir a los miembros de un grupo u organización (Codina, 2017); los cuales siguen al líder de forma voluntaria en la búsqueda del éxito y en la consecución de unos objetivos. Se han realizado extensos estudios sobre la materia y los investigadores han diferenciado varios tipos de liderazgo, siendo el transformacional uno de los que más atención ha recibido. En la actualidad, el liderazgo y el rol de ser jefe se confunden constantemente. El ocupar un rol de jefe en una empresa implica autoridad, donde los empleados siguen órdenes específicas y sus tareas se fundamentan en instrucciones estipuladas (Katzenbach \& Smith, 1996). El ambiente con jefatura rígida es frío y amargo, y por lo general, la relación entre los empleados y el jefe se limita a temas específicos de trabajo causando distancia e inseguridad

\section{El liderazgo transformacional y su influencia en las empresas}

En la actualidad existen muchos estudios realizados sobre el liderazgo y, su influencia en el desarrollo de habilidades del talento humano, existen autores, dichos estudios están basados en cuatro enfoques que describen las características esenciales de un líder. En la Teoría de los Rasgos, se puede enumerar algunas características genéricas de "personalidad, inteligencia, aptitudes y habilidades que poseen los líderes" (Villacreces, 2013). Así también existen las Teorías Conductuales que basan su estudio en los en la revisión de personalidades concretas de buenos líderes en contraste con líderes que carecen de efectividad (Villacreces, 2013). Se puede añadir al estudio la Teoría de las Contingencias se basa en los factores situacionales que hacen que un estilo conductual de "líder sea efectivo en una circunstancia determinada".

Adicionalmente existe el cuarto enfoque basado en el presente trabajo de investigación, llamado la Teoría del Liderazgo Transformacional, en el cual se explica la forma en que: 
"un líder motiva a sus seguidores a lograr un alto desempeño ayudándolos a encontrar y explotar sus propias habilidades con el objeto de cumplir las metas del negocio" (Villacreces, 2013)

Continuando con la línea de investigación analizamos el caso de la Empresa Sufrimiento S.A. en la cual se muestra cómo es el liderazgo en las empresas, el cual es considerado como una "estrategia o valor agregado para generar competitividad teniendo como principal objetivo orientar el diseño de programas de entrenamiento y desarrollo gerencial” (Codina, 2017).

Además, al analizar las características y rol del líder, se identifican varios personajes que participan el, los cuales en conjunto son las personas que intervienen directamente en la actividad y el desarrollo de la organización, en este caso vemos como se desenvuelve un equipo al empujar una carreta sobre un camino, donde existen integrantes como el Productor, el Hacedor altamente eficiente, el Hacedor menos eficiente, el Fuerte potencial de problemas y, la Persona Supresiva. Cada personaje tiene una característica y comportamiento que influye sobre el resultado de la actividad, adicional estableceremos la actuación del líder sobre el equipo, lo detallamos a continuación:

1. El Productor o puede ser llamado líder que sabe a dónde ir, que jala la carreta, él sabe que es lo que tiene que hacer y realmente emprendedor, toma plena responsabilidad por su trabajo y así aporta a la organización.

2. El Hacedor altamente eficiente: es el miembro que empuja la carreta junto al ritmo de su líder, tiene una inmensa voluntad para trabajar, aprender y mejorar, así también con una buena orientación y entrenamiento serelará un futuro Productor.

3. El Hacedor menos eficiente: es el miembro del equipo que está sentado sobre la carreta, tiene algo de voluntad para ayudar, sin embargo, su contribución no hace mucha diferencia en el resultado final.

4. El Fuerte Potencial de Problemas: es la persona que está sobre la rueda, su rendimiento como la ilustración sube y baja, alterna entre ayudar y destruir, su influencia hace el trabajo más difícil y menos predecible para los demás.

5. La Persona Supresiva: es el integrante que está deteniendo el avance de la carreta, es aquel que intenta constantemente destruir la operación, ocultando a menudo sus actos bajo una máscara de social "amable"; es importante destacar que si no son detectados a tiempo pueden convertirse en una amenaza muy peligrosa para cualquier organización.

Lo destacable del caso es que el líder debe aprender a diferenciar entre personas productivas y destructivas, debe saber disponer y coordinar el trabajo de todos los miembros del equipo, para así transformarlo en un grupo de trabajo de alto rendimiento, estable y productivo. (Camargo, 2014)

Debido a la notable competitividad en los mercados, los colaboradores pueden convertirse en la ventaja competitiva de cada organización, sobre todo si trabajan conjuntamente. Con esta actividad, el trabajo en equipo es una oportunidad para que el líder transformacional motive a los participantes del grupo a explotar y compartir sus habilidades (Palomo, 2013) 
Continuando con el estudio se han realizado diversas investigaciones sobre la materia y los investigadores han diferenciado varios tipos de liderazgo, siendo el transformacional uno de los que más atención ha recibido, su concepto fue originado e introducido por el experto en liderazgo James McGregor Burns. Éste lo definió como el tipo de liderazgo ostentado por aquellos individuos con una fuerte visión y personalidad, gracias a la cual son capaces de cambiar las expectativas, percepciones y motivaciones (Jiménez, 2014), así como liderar el cambio dentro de una organización. Asimismo, determinó que tal tipología de liderazgo era observable "cuando los líderes y seguidores trabajan juntos para avanzar a un nivel superior de moral y motivación" (Vásquez, 2013). Posteriormente fue el investigador Bernard M. Bass quién desarrolló la Teoría del Liderazgo Transformacional de Bass, cuya aportación es determinar el impacto que tiene el líder sobre los seguidores, y la reacción de ellos que está basada en la confianza, el respeto y la admiración de los mismos (Vásquez, 2013)

Entonces ¿cuál debería ser el rol del líder y cuáles podrían ser las características más importantes que debería poseer?

Siguiendo el estudio del modelo de Bass analizamos los componentes que tiene el liderazgo transformacional, se encuentran los siguientes:

1. Estimulación intelectual: el líder transformacional no se limita a desafiar el status quo dentro de una organización, sino que fomenta de forma intensiva la creatividad entre sus seguidores, alentando siempre a su equipo a explorar nuevas formas de hacer las cosas y nuevas oportunidades.

2. Consideración individualizada: el liderazgo transformacional es mantener líneas de comunicación abiertas con los seguidores, ya sea de forma individual como colectiva. De este modo se asegura que se compartan nuevas ideas, pudiendo surgir así productos o innovaciones. Son estos canales de comunicación los que permiten a los líderes un reconocimiento directo a sus seguidores, motivándolos y fomentando la proactividad.

3. Inspiración y motivación: gracias a su visión clara, los líderes transformacionales tienen la capacidad de articular a sus seguidores. Así el líder logra transmitir su motivación y pasión, lo que conduce a empleados con mayor proactividad y comprometidos con la organización.

4. Influencia idealizada: el líder transformacional se erige como un modelo para sus seguidores. Estos quieren emularlo como consecuencia de la confianza y respeto que tienen depositados en él. Es así que surgen nuevos líderes transformacionales dentro de la organización ya que el liderazgo es una capacidad innata, que puede desarrollarse y ser entrenada.

Es importante acotar los tipos de liderazgo según el investigador Burns (1978), describe tres tipos de líderes transformacionales en el contexto social, aunque no los reconoce como categorías (Vásquez, 2013), entre ellos tenemos:

- Liderazgo intelectual: es el que trabaja con ideas analíticas y normativas. No se desvincula de su contexto social. Tiene una conciencia de propósito. 
- Liderazgo reformador: cuenta con habilidades políticas excepcionales. Debe ser el líder un estratega y proclive a las alianzas. Es un liderazgo moral, que tiene gran poder de negociación.

- Liderazgo revolucionario: es el que encabeza una transformación de todo un sistema social. Es un líder totalmente dedicado a la causa, que puede llegar hasta ser mártir. Esto genera una conciencia social y política entre los líderes y los seguidores

Luego de revisar estas características, se puede comprender que el liderazgo transformacional es el de mayor aporte a las organizaciones, en las cuales sus objetivos sean fomentar la creatividad y la innovación entre sus empleados para obtener mayor competitividad (Vásquez, 2013)

\section{El líder transformacional y la gestión del cambio}

Los modelos de liderazgo orientados hacia el cambio en la actualidad han adquirido gran relevancia, esto se debe a las grandes transformaciones que hoy se ven en los ámbitos político y organizacional (García, 2011), la relevancia de estos modelos que sustentan el cambio, radica en que se debe realizar la creación de un nuevo sistema organizacional y su institucionalización, a través de nuevas formas de administración.

Es importante acotar que la gestión del cambio depende en gran medida del liderazgo, se puede establecer a través del siguiente proceso una influencia del liderazgo sobre la gestión del cambio, así tenemos lo siguiente:

a) Énfasis organizacional en la eficiencia y la adaptación, b) dominancia del núcleo técnico y unidades organizacionales, c) estructura organizacional y d) forma de gobierno.

A través del estudio del autor se puede establecer la correlación entre los factores y dos tipos de líder transformacional, determinándose lo siguiente:

- Proveedores de responsabilidades y prioridades claras, en un marco de comunicación constante y libertad para la improvisación. Los líderes crean estructuras que promueven la creatividad de sus seguidores

- Exploradores del futuro mediante la experimentación, con una gran variedad de pruebas de bajo costo, lo cual permite el aprendizaje organizacional.

- Vinculadores del presente con el futuro, con la secuencia de los proyectos y procedimientos transicionales poco a poco se van haciendo familiares para todos los miembros de la organización.

El líder transformacional provee de cambio y movimiento a la organización; por ello debe poseer una visión de las metas a alcanzar para guiar a la institución en nuevas direcciones. Este tipo de líder las nuevas posibilidades y promueve una visión de futuro porque es guiado por un fuerte sentido de propósito (Vásquez, 2013) El cambio que promueve el líder debe estar por encima de la rutina de los sistemas mecánicos orientados al poder. Los autores sostienen que esto sucede porque el líder transformacional cuestiona todo y promueve un estilo de pensamiento no tradicional (Vásquez, 2013). 
Es así, que existe una relación muy cercana entre el líder y lo que busca de su equipo, el desarrollo de las capacidades de los miembros del staff, siguen siendo responsabilidad del líder (Uribe \& Molina, 2013), que busca el óptimo desempeño y la búsqueda de llegar a los resultados.

Siguiendo la línea de investigación se analiza el equipo de alto rendimiento como propuesta de desarrollo de las empresas, que buscan la eficiencia y que su talento humano busca colaborar activamente con la organización

Entre los principales aspectos de influencia del liderazgo transformacional es el desarrollo de equipos de alto rendimiento, los cuales sus integrantes buscan en compañía del líder la participación activa en la consecución de resultados. Los integrantes del equipo se vuelven protagonistas en la búsqueda de la solución al problema (Palomo, 2013)

Continuando con el análisis de la investigación se encuentra los siguientes conceptos a desarrollar:

\section{Desarrollo de equipos de alto rendimiento}

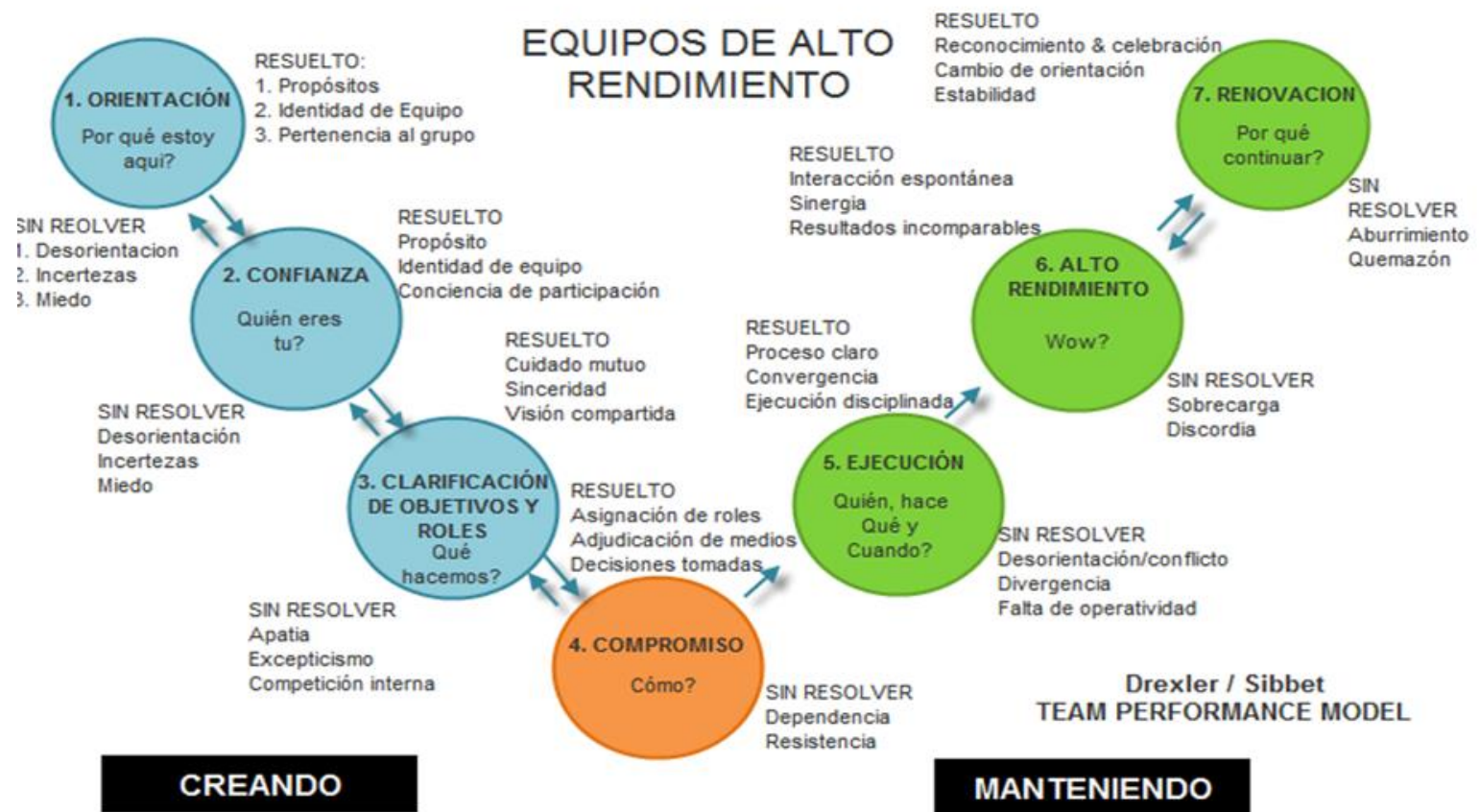

Imagen 1. Modelo de desarrollo de equipos de alto rendimiento Fuente: A personality trait based interactionist model of job performance Tett, Robert $P$.

En la imagen 1 podemos analizar las etapas que se desarrolla para la búsqueda de un equipo de alto rendimiento. Este proceso establece según las etapas: Esencial, emocional, mental y físico. Existe un análisis de preguntas que ayudan al equipo ¿Por qué estamos juntos? y la otra pregunta ¿Seguimos?; existe el proceso de la Intención, Química, Información, Implementar, el Feedback, el alto rendimiento y la etapa de renovar (Tett \& Burnett, 2003). 
Entre las características que debe tener un equipo de alto desempeño se encuentran las siguientes:

- Objetivos y metas que le dan identidad al equipo

- Ambiente de trabajo

- Solución de conflictos

- Planes de aprendizaje y formación permanentes

- Liderazgo

- Toma de decisiones

- Comunicación eficaz

- Valores y creencias comunes

- Relaciones con el medio (interno y externo)

- Importancia de los resultados y del reconocimiento a miembros del equipo

\section{Diferencias entre equipo de trabajo y equipos de alto desempeño}

Existen diferencias entre los equipos de trabajo y los equipos de alto desempeño.

\begin{tabular}{|c|c|}
\hline EQUIPO DE TRABAJO & EQUIPOS DE ALTO DESEMPENO \\
\hline $\begin{array}{c}\text { Tienen un objetivo en común } \\
\text { compartido y se sienten } \\
\text { corres ponsables }\end{array}$ & $\begin{array}{c}\text { Adicionamente se conectan con el } \\
\text { propós ito tras cendente }\end{array}$ \\
$\begin{array}{c}\text { El equipo responde en conjunto de1 } \\
\text { trabajo realizado }\end{array}$ & $\begin{array}{c}\text { El trabajo técnico es de excelencia y } \\
\text { adicionalmente bus can generar } \\
\text { convivencias de excelencia }\end{array}$ \\
\hline $\begin{array}{c}\text { Sus miembros complementan sus } \\
\text { talentos y fortalezas }\end{array}$ & $\begin{array}{c}\text { Sus miembros han des arrollado } \\
\text { dinámicas que hacen des pegar } \\
\text { talentos y fortalecimiento mutuo }\end{array}$ \\
\hline $\begin{array}{c}\text { Es necs aria la coordinación entre } \\
\text { todos y exige altos estándares de } \\
\text { comunicación }\end{array}$ & $\begin{array}{c}\text { No sólo funcionan los as pectos de } \\
\text { coordinación, sino adicionalmente } \\
\text { des arrollan la conectividad }\end{array}$ \\
\hline
\end{tabular}

Imagen 2. Diferencias entre equipo de trabajo y equipos de alto desempeño Fuente: Elaboración propia

En la Imagen 2 observamos las diferencias entre los equipos de trabajo y los equipos de alto desempeño, es importante añadir que el involucrarse en la realidad del equipo, no solo con la participación, sino también con el auto liderar el grupo, es una marcada diferencia en la consecución de los objetivos. El tener un objetivo claro y retador y estar estructurados en función de los resultados que se esperan, es contar con miembros competentes (Balda \& Guzmán, 2015), tener un compromiso común, operar dentro de un clima de cooperación, contar con parámetros para la medición de su desempeño, recibir apoyo y reconocimiento externo, a la vez que tienen 
un liderazgo basado en los principios y valores de la organización a la que pertenecen (Tett \& Burnett, 2003)

Siguiendo el estudio podemos analizar el ¿por qué de los equipos?, y la importancia que éste tiene como herramienta de impulso en las instituciones que buscan un desarrollo en el futuro, y que buscan la calidad y el servicio.

\section{Aportes de un equipo de alto desempeño}

Existen diversos estudios sobre el presente tema de investigación, entre los principales puntos que encontramos tenemos los siguientes:

- Ampliar las ideas aportadas por otros, con ejemplos precisos

- Asumir el liderazgo para conducir al equipo en momentos de tensión, stress o pérdida de foco y tiempo

- Evidenciar capacidad de trabajo y de ejecución, involucrándose voluntariamente en el hacer

- Formular preguntas para que se clarifique la información, para evidenciar lo que no está claro, o para identificar la información que falta

A través de los estudios encontramos la relación entre el liderazgo y el desarrollo de los equipos de alto desempeño, existe un capítulo importante como la Inteligencia Emocional como "el potencial para aprender las habilidades prácticas que se basan en sus cinco elementos: conocimiento de uno mismo, motivación, autorregulación, empatía y destreza para las relaciones" (Ortega, 2014)

La inteligencia emocional que posee el líder interviene directamente en la motivación de su equipo, adicional es importante analizar la proporción de ese potencial pueda ser traducido a las facultades que aplica su equipo en las actividades laborales (Ortega, 2014). La Inteligencia Emocional afecta a la forma de gestionar el comportamiento, a la forma de afrontar las relaciones sociales, y tomar decisiones personales. La Programación Neuro Lingüística conocida como "un conjunto de sistemas para entender y programar nuestro comportamiento" (Ortega, 2014). Se trata de un conjunto de herramientas que permite analizar a la persona y crear la visión que el líder desea transmitir.

\section{Dirección y liderazgo situacional de equipos}

Un equipo de alto desempeño debe tener claro la idea del líder, y que según su forma de liderazgo comparte o toma decisiones en beneficio del grupo, entonces ¿Cuáles deberían ser los indicadores que debe conocer un equipo de alto desempeño?

Entre los principales puntos tenemos:

- Propósito y Valores: es el sentido del propósito que se comparte en conjunto con todos los miembros del equipo, que tiene una visión convincente y valores que soportan este desarrollo. 
- Empoderamiento: La confianza del equipo en superar obstáculos está basado en la capacidad para lograrlo. Existe autoridad para tomar decisiones y actuar, elegir con fronteras claras, así también se busca autonomía con oportunidades.

- Relaciones y comunicación: los miembros del equipo asumen y comparten riesgos, a través de la sociabilización de pensamientos, opiniones y sentimientos sin temor.

- Flexibilidad: todos los miembros del equipo son responsables del desempeño, el desarrollo y el liderazgo.

- Óptima productividad: todos asumen su rol y se enorgullecen de los logros del equipo, aportando con calidad y cantidad de trabajo que se requiera.

- Moral: es el resultado de todo lo anterior.

Es importante la influencia del liderazgo transformacional en el desarrollo de equipos de alto desempeño, "los líderes al modelar su equipo, transmiten a los integrantes la visión, coordinan la conformación del grupo" (Moreira, 2010), buscan el desarrollo de los talentos de los miembros de su equipo y acompaña en el proceso de la consecución de resultados. La Relación entre el liderazgo transformacional y el desarrollo de equipos de alto rendimiento en las empresas, influyen sobre los resultados y la consecución de objetivos (Uribe \& Barbosa, 2013).

Después del estudio realizado a continuación se enumera las siguientes Conclusiones:

- La influencia del líder sobre el equipo genera un ambiente de visión compartida, que establece roles de trabajo coordinados y con decisiones compartidas. El líder es democrático o autocrático, según su estilo de mando interviene directamente en el rendimiento de los equipos.

- En el desarrollo de equipos de alto rendimiento existe una alta credibilidad y confianza entre todos los miembros del equipo, donde existe una exposición de las ideas con claridad y con la aplicación de competencias fundamentales.

- El líder transmite claramente el propósito y visión, así también establece un vínculo muy cercano con su equipo de trabajo basado en responsabilidad mutua y establece resultados con indicadores basados en alto desempeño.

\section{Bibliografía}

Balda, R., \& Guzmán, A. (2015). Liderazgo Educativo Transformacional Como Necesidad De Las Instituciones Educativas En La República De Ecuador. Portoviejo: Repositorio Universidad Laica Eloy Alfaro De Manabí.

Camargo, D. (2014). Equipos De Alto Rendimiento. Bogotá: Repositorio Institucional Universidad Militar Nueva Granada.

Codina, A. (2017). 10 Habilidades Directivas. ¿Por Qué? ¿Para Qué? ¿Cómo? La Habana: Infodir - Issn 1996.

García, M. (2011). Liderazgo Transformacional Y La Facilitación De La Aceptación Al Cambio Organizacional. Pensamiento Psicológico - Issn 1657-8961, 12. 
Jiménez, C. (2014). Relación Entre El Liderazgo Transformacional De Los Directores Y La Motivación Hacia El Trabajo Y El Desempeño De Docentes De Una Universidad Privada. Bogotá: Repositorio Universidad Católica De Colombia.

Katzenbach, J., \& Smith, D. (1996). Sabiduría De Los Equipos. Madrid: Ediciones Diaz De Santos S.A.

Moreira, C. (2010). Liderazgo Transformacional Y Género En Organizaciones Militares. Madrid: Repositorio Universidad Complutense De Madrid.

Nader, M., \& Castro, A. (2007). Influencia De Los Valores Sobre Los Estilos De Liderazgo: Un Análisis Según El Modelo De Liderazgo Transformacional - Transaccional De Bas. Universidad Piscología Issn 1657-9267, 17.

Ortega, E. (2014). Inteligencia Emocional En Las Organizaciones. Guadalajara: Repositorio Universidad De Guadalajara - Centro Universitario De La Costa.

Palomo, M. (2013). Liderazgo Y Motivación De Equipos De Trabajo. Madrid: Esic Editorial.

Pico, L. (2016). El Talento Humano Calificado Como Recurso Indispensable Para La Organización. Innova Research Journal - ISSN 2477-9024, 8.

Tett, R. P., \& Burnett, D. D. (2003). A Personality Trait Based Interactionist Model of Job Performance. Apa Psycnet, 12.

Uribe, A., \& Barbosa, D. (2013). Universidad Empresa, 23.

Uribe, A., \& Molina, J. (2013). Rosario: Repositorio Universidad Del Rosario.

Vásquez, A. (2013). Interdependencia Entre El Liderazgo Transformacional, Cultura Organizacional Y Cambio Educativo: Una Reflexión. Revista Iberoamericana Sobre Calidad, Eficacia Y Cambio En Educación - Issn: 1696-4713, 19.

Villacreces, A. (2013). Efectos Del Liderazgo Transformacional En Las Empresas. Quito: Repositorio Digital Universidad San Francisco De Quito - Colegio De Administración Para El Desarrollo. 